\title{
Publisher Correction: Parent of origin genetic effects on methylation in humans are common and influence complex trait variation
}

Yanni Zeng ${ }^{1}$, Carmen Amador (10 1, Charley Xia ${ }^{1,2}$, Riccardo Marioni ${ }^{3,4}$, Duncan Sproul (1) 1,5, Rosie M. Walker ${ }^{3,4}$, Stewart W. Morris ${ }^{4}$, Andrew Bretherick1, Oriol Canela-Xandri1,2, Thibaud S. Boutin (1) ${ }^{1}$, David W. Clark ${ }^{6}$, Archie Campbell (1D 4, Konrad Rawlik (D) 2, Caroline Hayward (1) 1, Reka Nagy ${ }^{1}$, Albert Tenesa (i) 1,2, David J. Porteous (10) 3,4, James F. Wilson (1) 1,6, Ian J. Deary 3,7, Kathryn L. Evans ${ }^{3,4}$, Andrew M. Mclntosh (1D 3,8, Pau Navarro (1) ${ }^{1} \&$ Chris S. Haley (i) ${ }^{1,2}$

Correction to: Nature Communications https://doi.org/10.1038/s41467-019-09301-y, published online 27 March 2019.

In the original version of this Article, the legend in the upper panel of Fig. 2 incorrectly read 'paternal imprinting' and should have read 'maternal imprinting'. This has been corrected in both the PDF and HTML versions of the Article.

Published online: 01 May 2019

Open Access This article is licensed under a Creative Commons Attribution 4.0 International License, which permits use, sharing, adaptation, distribution and reproduction in any medium or format, as long as you give appropriate credit to the original author(s) and the source, provide a link to the Creative Commons license,
and indicate if changes were made. The images or other third party material in this article are included in the article's Creative Commons license, unless indicated otherwise in a credit line to the material. If material is not included in the article's Creative Commons license and your intended use is not permitted by statutory regulation or exceeds the permitted use, you will need to obtain permission directly from the copyright holder. To view a copy of this license, visit http://creativecommons.org/licenses/by/4.0/.

(c) The Author(s) 2019

\footnotetext{
${ }^{1}$ MRC Human Genetic Unit, Institute of Genetics and Molecular Medicine, University of Edinburgh, Edinburgh EH4 2XU, UK. ${ }^{2}$ The Roslin Institute and Royal (Dick) School of Veterinary Sciences, University of Edinburgh, Edinburgh EH25 9RG, UK. ${ }^{3}$ Centre for Cognitive Ageing and Cognitive Epidemiology, University of Edinburgh, Edinburgh EH8 9JZ, UK. ${ }^{4}$ Centre for Genomic and Experimental Medicine, IGMM, University of Edinburgh, Edinburgh EH4 2XU, UK. ${ }^{5}$ Edinburgh Cancer Research Centre, Institute of Genetics and Molecular Medicine, University of Edinburgh, Edinburgh EH4 2XR, UK. ${ }^{6}$ Centre for Global Health Research, Usher Institute of Population Health Sciences and Informatics, University of Edinburgh, Edinburgh EH8 9AG, UK. ${ }^{7}$ Department of Psychology, University of Edinburgh, Edinburgh EH8 9JZ, UK. ${ }^{8}$ Division of Psychiatry, University of Edinburgh, Edinburgh EH10 5HF, UK. Correspondence and requests for materials should be addressed to C.S.H. (email: chris.haley@igmm.ed.ac.uk)
} 